\title{
Short communication: Anesthetic residues in milk after topical application during treatment of hoof lesions in dairy cows
}

\author{
G. Stilwell, * A. M. Ferrador, (1) M. S. Santos, and J. M. Domingues (1) \\ Animal Behavior and Welfare Laboratory, Center of Interdisciplinary Research in Animal Health, Faculty of Veterinary Medicine, Lisbon University, \\ Portugal 1300-477
}

\section{ABSTRACT}

Hoof lesions in dairy cows are usually treated by trimming the hoof. However, trimming by itself can cause severe pain or exacerbate already existing pain. Hoof trimming is usually not carried out by trained veterinarians, and pain management is not provided. Pain control during trimming is not only an ethical obligation but also allows for better manipulation and more meticulous treatment. Tri-Solfen (Bayer Animal Health, Pymble, Australia) is a spray gel containing lidocaine, bupivacaine, and cetrimide that is easily applied topically and has demonstrated pain-mitigation effects during and after hoof trimming. In the European Union, these local anesthetics are not approved for use in food-producing animals because of a lack of residue data and concerns about genotoxic effects in cattle and humans. The aim of this study was to assess lidocaine, bupivacaine, and 2,6-xylidine residues in milk after TriSolfen application in dairy cows. Five dairy cattle in the dry-off period were enrolled in the study based on clinical evidence of lameness (score $\geq 3$ on a 5 -point scale). After cleaning and superficial trimming, we applied 3 to $14 \mathrm{~mL}$ of Tri-Solfen to the lesions before continuing treatment. Two milk samples were collected per animal in the following 4 milkings and analyzed in a reference laboratory. Residues of lidocaine above the limits of quantification $(0.2 \mu \mathrm{g} / \mathrm{L})$ were found in milk samples in the first milking $6 \mathrm{~h}$ after treatment in only 2 cows. This study shows that excretion of local anesthetics and their metabolites in milk after topical application of Tri-Solfen is negligible and even undetectable after the first milking $6 \mathrm{~h}$ post-treatment.

Key words: hoof disease, pain management, animal welfare, local anesthesia, anesthetic residue

Received June 25, 2019.

Accepted August 25, 2019.

*Corresponding author: stilwell@fmv.ulisboa.pt

\section{Short Communication}

Hoof lesions in dairy cows are treated by trimming the hoof, a treatment that may exacerbate already existing pain. Worldwide, hoof trimming is usually carried out by non-veterinarians and without pain management (Becker et al., 2014). Identifying effective and safe treatment options for managing pain during hoof trimming is critical for improving dairy cattle welfare.

Recently, a gel-based topical local anesthetic (TriSolfen; Bayer Animal Health, Pymble, Australia) demonstrated rapid anesthesia when applied to castration wounds in calves, with analgesia that lasted $24 \mathrm{~h}$ (Lomax and Windsor, 2013). In addition, a recent study demonstrated that this product could mitigate pain when applied before trimming injured/diseased hooves in dairy cattle (Stilwell et al., 2019).

One of the metabolites of lidocaine is 2,6-xylidine. Current research suggests that at high doses, 2,6-xylidine may have mutagenic or genotoxic potential (Chao et al., 2012), although findings have been inconsistent (Kirkland et al., 2012). In cattle, 2,6-xylidine has been detected in plasma, urine, milk, and tissues after intramuscular administration of lidocaine (Hoogenboom et al., 2015). Using residue depletion data from these studies, the European Medicines Agency (EMA) has recommended a withholding period of $28 \mathrm{~d}$ for meat and $15 \mathrm{~d}$ for milk following the injected use of lidocaine in cattle (EMA, 2015). Currently, in the European Union, lidocaine can be applied only within the so-called Cascade rules (EMA, 1999), a legislative provision that allows a veterinary surgeon to prescribe unauthorized medicines under certain conditions (https://assets.publishing .service.gov.uk/government/uploads/system/uploads/ attachment_data/file/424668/VMGNote13.PDF). However, lidocaine is commonly used in the United States, Canada, Australia, New Zealand, and other countries, with meat and milk withdrawal periods of only a few days. No research to date has evaluated the maximum residue limit of bupivacaine, although one study has suggested that bupivacaine does not metabolize to 2,6-xylidine in cattle and sheep (Edwards et al., 2017). 
The aim of this study was to evaluate the concentration of lidocaine, bupivacaine, and 2,6-xylidine residues in milk after the topical application of Tri-Solfen to hoof lesions.

All procedures were approved under the Portuguese Directorate-General for Food and Veterinary, no. 49/ ECVPT/2017, and the Ethics and Animal Welfare Committee of the Faculty of Veterinary Medicine at the University of Lisbon, Ref. 003/2018, and accepted by the animals' owners.

Animals included in this study belonged to 2 dairy herds with 200 and 800 milking cows, respectively, kept year-round in freestall stables with zero grazing. The inclusion criterion for the study was lame cows presenting with hoof lesions (e.g., sole ulcers, toe ulcers, white line disease, interdigital necrobacillosis, or interdigital/digital dermatitis). Cows were identified as being clinically lame (a score of 3 or higher on a 5-point scale) by the farms' veterinarians. All cows were being dried off; no animal destined to produce milk for human consumption in the following $60 \mathrm{~d}$ was included. Treatment was done with a commercially available topical anesthetic, hemostatic, and antiseptic formulation for the alleviation of pain in production animals that contained lidocaine, bupivacaine, adrenaline, and cetrimide in a gel base (Tri-Solfen).

Five lame cows were included in the study (Table 1 ). Lesions (1 interdigital necrobacillosis, 1 toe ulcer, and 3 sole ulcers, each in different stages) were described, classified, and graded according to dimension. The lesion area was then trimmed and enough Tri-Solfen was applied to cover the wound once the corium was exposed. The quantity of Tri-Solfen used (in $\mathrm{mL}$ ) was recorded. Two samples of milk $(10 \mathrm{~mL})$ from each cow were collected on each of the subsequent 4 milkings (6, 18, 30, and $42 \mathrm{~h}$ post-treatment), immediately frozen, and sent at $-70^{\circ} \mathrm{C}$ to a reference laboratory in Australia (Eurofins Agroscience Testing Pty Ltd., Lane Cove West, Australia). All 40 samples (2 samples per cow per milking) were analyzed for concentrations of lidocaine and its metabolites (monoethylglycinexylidide, glycinexylidide, 3-hydroxy lignocaine, 2,6-xylidine), bupivacaine and its metabolites (110 N-desbutyl bupivacaine, 3-hydroxybupivacaine), and cetrimide. Specimen extraction and determination of residues were performed according to a method developed at the analytical test site based on a published paper (Edwards et al., 2017). Quantitation was performed using liquid chromatography with tandem MS detection of lignocaine, bupivacaine, and metabolites. The limit of detection (LOD) and limit of quantitation (LOQ) for this method were $0.07 \mu \mathrm{g} / \mathrm{L}$ and $0.2 \mu \mathrm{g} / \mathrm{L}$, respectively, for lignocaine, bupivacaine, and all measured metabolites, including 2,6-xylidine. For cetrimide, the LOD and LOQ were set at $3 \mu \mathrm{g} / \mathrm{L}$ and $10 \mu \mathrm{g} / \mathrm{L}$, respectively. This method, previously developed and fully validated to Veterinary International Conference on Harmonization guidelines (https://www.vichsec.org/en/guidelines .html) as part of related metabolism studies in bovine tissues, was similarly validated as part of the present milk study. The efficiency of the method was monitored by analyzing control (untreated) samples and fortified control (recovery) samples concurrently with the test samples.

Table 1 lists all detected residues found per animal, indicating the milking at which they were found. Because the maximum residue limit has not been established for these drugs and their metabolites, we used the LOD to determine the presence of residues. Accuracy and precision could not be verified for values below the LOQ and, therefore, should not be considered as relevant. In all of the samples analyzed, 2,6-xylidine was below the LOQ and was thus unquantifiable.

In cow \#4423, lidocaine residues were detected under LOQ values at the first milking (6 h after application) in both samples and bupivacaine residues were detected above the LOQ at the second milking (18 $\mathrm{h}$ after application), but in only 1 sample. In cow \#4460, the lidocaine metabolite monoethylglycinexylidide was detected above the LOQ at the second milking ( $18 \mathrm{~h}$ after application), but in only 1 sample. In cow \#4828, the metabolite $N$-desbutyl bupivacaine was detected above LOQ values at the fourth milking (42 $\mathrm{h}$ after application), but in only 1 of the samples. The very low levels detected, which may be variably present in milk, as well as the poor accuracy and precision at levels below the LOQ, are the most reasonable explanations why these 3 animals had only 1 samples above the LOQ when the others were under the LOD.

In in cow \#395 and cow \#9071, lidocaine residues were detected above LOQ values in both samples taken at $6 \mathrm{~h}$ after application, but all were very low $(<0.5$ $\mu \mathrm{g} / \mathrm{L}$ ). In cow \#395, lidocaine residues were again detected at the third milking (approximately $30 \mathrm{~h}$ after application), but only in 1 sample and below the LOQ threshold. Bupivacaine and its metabolites were also detected below LOQ values in 1 sample from the first milking in cow \#395 and cow \#9071.

These 2 cows showed consistent values at $6 \mathrm{~h}$, suggesting that very low excretion might occur in the first hours after treatment in some cases. Our values were much lower $(0.204$ to $0.398 \mu \mathrm{g} / \mathrm{L}$ vs. 14.5 to $66 \mu \mathrm{g} / \mathrm{L})$ than those found in other studies after parenteral injection (Puente and Josephy, 2001), and were found in only 2 of the 5 cows treated. This finding may indicate limited absorption following topical application, and we suggest that the presence of residues in milk after topical application deserves further investigation. Detected 


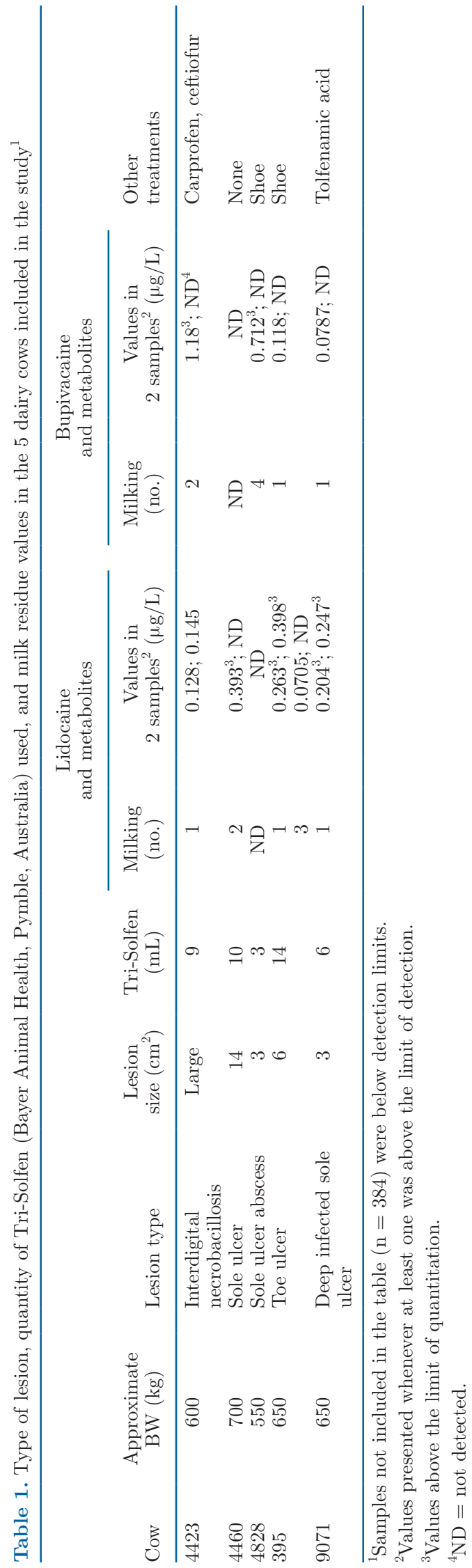

residues could have occurred because of the volume of Tri-Solfen applied to the lesion [the largest quantity of Tri-Solfen used (14 mL) was in cow \#395], the type or extent of the lesion (cow \#9071 had a deep sole ulcer), or simply because of individual biological differences. Only by increasing the number of treated animals will it be possible to confirm these findings.

No studies have been published determining the limits for lidocaine, bupivacaine, and their metabolites in milk after topical application and a subsequent withdrawal period. A study evaluating the pharmacokinetics of lidocaine in mature Holstein cows following an inverted L nerve block suggested a milk withdrawal period of 72 $\mathrm{h}$, because detectable lidocaine concentration in milk was present at $48 \mathrm{~h}$ but not at $60 \mathrm{~h}$ post-injection (Sellers et al., 2009). Hoogenboom et al. (2015) suggested 7 and $28 \mathrm{~d}$ as off-label withdrawal times for milk and meat, respectively, after subcutaneous and intramuscular injections. However, these studies can only assist as guidelines, because topical application and local injection of lidocaine will certainly have very different pharmacokinetics.

It is important to highlight the fact that no sample showed any quantifiable trace of 2,6-xylidine. Because this compound is highly reactive with lactose and glucose (EMA, 2015), it is unknown how much of the amount excreted into milk is deactivated via chemical reactions in the milk.

Cetrimide was detected in milk from cows \#395 (3.48 $\mu \mathrm{g} / \mathrm{L})$ and \#4423 $(3.66 \mu \mathrm{g} / \mathrm{L})$ in 1 sample from the second milking (18 h after application) and in the 2 samples from the first milking of cow \#9071 (11.5 $\mu \mathrm{g} / \mathrm{L}$ and $24.4 \mu \mathrm{g} / \mathrm{L}$; results not shown).

According to the LOQ established by Eurofins Agroscience Testing Pty Ltd., no significant milk residues of lidocaine, bupivacaine, their metabolites, or cetrimide were found in any of the 5 cows in the first milking $6 \mathrm{~h}$ after application. These findings suggest that the application of the topical anesthetic on hoof lesions in dairy cows would not lead to a significant presence of any of the product components or its metabolites in milk $6 \mathrm{~h}$ post-treatment. Because these were cows being dried off and milked twice a day, we cannot be sure that results would be comparable in high-yielding animals or in cows being milked more frequently. We emphasize that these are preliminary results that need confirmation.

Avoiding drug residues in food-producing animals is a major worldwide concern. Although our study indicates that applying lidocaine and bupivacaine when trimming hoof lesions in dairy cows will result in very low levels of residues in milk for a very short time, suggesting minimal absorption when applied topically, more studies in a larger set of animals should follow. 
These would clarify if withdrawal periods are overestimated and need to be reviewed without risking food safety.

\section{ACKNOWLEDGMENTS}

We acknowledge the funding by Project UID/ CVT/00276/2019 (CIISA) and the help from Animal Ethics Pty Ltd. The authors also thank the farmers for their participation in this study.

\section{REFERENCES}

Becker, J., M. Reist, and A. Steiner. 2014. Factors influencing the attitudes of cattle veterinarians, farmers, and claw trimmers towards the pain associated with the treatment of sole ulcers and the sensitivity to pain of dairy cows. Vet. J. 200:38-43. https://doi.org/ 10.1016/j.tvjl.2014.01.016.

Chao, M. W., M. Y. Kim, W. Ye, J. Ge, L. J. Trudel, C. L. Belanger, P. L. Skipper, B. P. Engelward, S. R. Tannenbaum, and G. N. Wogan. 2012. Genotoxicity of 2,6-and 3,5 dimethylaniline in cultured mammalian cells: The role of reactive oxygen species. Toxicol. Sci. 130:48-59. https://doi.org/10.1093/toxsci/kfs229.

Edwards, S., A. Smith, and G. Doran. 2017. Pharmacokinetics, Residue Kinetics and Residue Depletion Studies for Lignocaine and Bupivacaine in Sheep and Cattle. Meat and Livestock Australia Ltd., North Sydney, Australia.

EMA (European Medicines Agency). 1999. Committee for medicinal products for veterinary use. Lidocaine. Summary report. EMEA/ MRL/584/99-FINAL. Accessed Jul. 30, 2019. http://www.ema .europa.eu/docs/en_GB/document_library/Maximum_Residue _Limits_Report/2009/11/WC500014745.pdf.

EMA (European Medicines Agency). 2015. CVMP assessment report regarding the request for an opinion under Article 30(3) of Regulation (EC) No 726/2004 in relation to the potential risk for the consumer resulting from the use of lidocaine in food producing species. EMA/CVMP/118717/2015. Accessed Jul. 30, 2019. https: //www.ema.europa.eu/en/documents/report/cvmp-assessment -report-regarding-request-opinion-under-article-303-regulation -ec-no-726/2004-relation-potential-risk-consumer-resulting-use -lidocaine-food-producing-spec_en.pdf.

Hoogenboom, R. L., T. Zuidema, M. L. Essers, A. M. van Vuuren, P. G. van Wikselaar, J. C. H. van Eijkeren, M. J. B. Mengelers, M. J. Zeilmaker, and A. S. Bulder. 2015. Concentrations of dimethylaniline and other metabolites in milk and tissues of dairy cows treated with lidocaine. Food Addit. Contam. Part A Chem. Anal. Control Expo. Risk Assess. 32:1256-1264. https://doi.org/ 10.1080/19440049.2015.1064172.

Kirkland, D., M. Ballantyne, S. Harlfinger, O. Will, U. Jahnel, A. Kraus, and C. V. Dorp. 2012. Further investigations into the genotoxicity of 2,6-xylidine and one of its key metabolites. Regul. Toxicol. Pharmacol. 62:151-159. https://doi.org/10.1016/j.yrtph.2011 .08 .010

Lomax, S., and P. A. Windsor. 2013. Topical anesthesia mitigates the pain of castration in beef calves. J. Anim. Sci. 91:4945-4952. https: //doi.org/10.2527/jas.2012-5984.

Puente, N. W., and P. D. Josephy. 2001. Analysis of the lidocaine metabolite 2,6-dimethylaniline in bovine and human milk. J. Anal. Toxicol. 25:711-715.

Sellers, G., H. C. Lin, M. G. Riddell, W. R. Ravis, S. H. Duran, and M. D. Givens. 2009. Pharmacokinetics of lidocaine in serum and milk of mature Holstein cows. J. Vet. Pharmacol. Ther. 32:446-450. https://doi.org/10.1111/j.1365-2885.2009.01061.x.

Stilwell, G. T., A. M. Ferrador, M. S. Santos, J. M. Domingues, and N. Carolino. 2019. Use of topical local anesthetics to control pain during treatment of hoof lesions in dairy cows. J. Dairy Sci. 102:63836390. https://doi.org/10.3168/jds.2018-15820.

\section{ORCIDS}

G. Stilwell ๑ https://orcid.org/0000-0003-3733-3223

A. M. Ferrador $\odot$ https://orcid.org/0000-0003-4811-1458

J. M. Domingues @ https://orcid.org/0000-0002-3955-8824 